\title{
Assistência ao parto no Município do Rio de Janeiro: perfil das maternidades e o acesso da clientela
}

\author{
Obstetric care during childbirth in Rio de Janeiro: \\ hospital practices and user access
}

Tatiana Pacheco Campos 1

Marilia Sá Carvalho 2

\footnotetext{
1 Gerência de Programas de Saúde da Criança, Coordenação de Programas de Atendimento Integral à Saúde, Superintendência de Saúde Coletiva, Secretaria Municipal de Saúde do Rio de Janeiro. Rua Afonso Cavalcanti 455 sala 823 , Rio de Janeiro, $R J$ 21040-210, Brasil.

2 Departamento de Epidemiologia e Métodos Quantitativos em Saúde, Escola Nacional de Saúde Pública, Fundação Oswaldo Cruz.

Rua Leopoldo Bulhões 1480, 8 o andar, Rio de Janeiro, $R J$ 21041-210, Brasil.
}

\begin{abstract}
The purpose of this paper is to identify and describe Rio de Janeiro maternity hospital profiles and the route between the mother's place of residence and the hospital. Data sources were: the State Live Birth Information System (1995) and the National Survey on Medical Care (1992). Two groups of maternity hospitals were identified using multivariate cluster analysis. Group A had an extremely high cesarean rate (81\%), with mothers and neonates presenting good health conditions. Cesarean rates were lower in Group B, although still high (32\%), and other variables reflected worse neonatal conditions. Cesarean rate was the indicator which best discriminated between the groups, followed by proportion of adolescent mothers and mothers with a high school education. The uneven spatial distribution of maternity hospitals, which were concentrated in the richest area of the city, was a factor in the long routes used by women to reach medical care for childbirth.
\end{abstract}

Key words Midwifery; Delivery; Health Services Accessibility

Resumo Neste trabalho analisou-se a assistência ao parto, caracterizando o perfil das principais maternidades e o deslocamento da clientela, ou seja, o fluxo entre residência e local de nascimento. Os indicadores utilizados foram construídos a partir do Sistema de Informação sobre Nascidos Vivos (SINASC) em 1995 e da Pesquisa sobre Assistência Médico-Sanitária (AMS), com dados para 1992. Através de classificação multivariada foram identificados dois tipos de maternidades: um com grande número de partos cesáreos, boas condições da parturiente e recém-nato; e outro com maior proporção de partos espontâneos e indicadores que apontam riscos do recém-nascido. As proporções de: mães com escolaridade igual ou superior ao ensino médio, mães adolescentes e partos cesáreos são os indicadores que melhor caracterizaram os grupos. Identificou-se grande heterogeneidade na distribuição espacial das maternidades, concentrados nas regiões mais ricas da cidade, determinando, conseqüentemente, longos trajetos das gestantes na busca da assistência ao parto.

Palavras-chave Tocologia; Parto; Acesso aos Serviços de Saúde 


\section{Introdução}

Este artigo visa descrever e classificar os perfis das principais maternidades do Município do Rio de Janeiro, utilizando análise de cluster multivariada sobre as variáveis da declaração de nascido vivo (DN) e integrando diferentes fontes de dados secundários e métodos de análise exploratória de dados. Buscou-se, também, identificar a relação entre oferta de serviços de saúde e fluxo de gestantes entre o local de residência e a maternidade, utilizando ferramentas de Sistema de Informações Geográficas (SIG).

O uso de dados secundários traz à baila a questão da qualidade e confiabilidade destes. Entretanto, dados de alta qualidade só estarão disponíveis quando forem intensamente utilizados, estimulando a melhoria de confiabilidade (Caper, 1987). Buscando estudar a relação entre perfil epidemiológico do recém-nascido e da gestante com a oferta de serviços de saúde, foram utilizados os bancos de dados do Sistema de Informações sobre Nascidos Vivos (SINASC) e da Pesquisa sobre Assistência MédicoSanitária (AMS).

A Pesquisa sobre Assistência Médico-Sanitária é realizada pela Fundação Instituto Brasileiro de Geografia e Estatística (IBGE), em convênio com o Ministério da Saúde, produzindo periodicamente a atualização cadastral de todos os estabelecimentos de saúde (públicos ou privados) quanto ao perfil assistencial, estrutura física, recursos humanos e produção de atividades. Segundo Médici (1992), sua cobertura está em torno de $90 \%$ dos serviços de saúde oferecidos no país.

Em recente estudo realizado sobre o Sistema de Informações sobre Nascidos Vivos, utilizando como unidade de análise os bairros do Município do Rio de Janeiro, foi evidenciado que os indicadores que melhor caracterizaram os bairros foram as proporções de: nascidos vivos com Apgar bom no primeiro minuto (entre 8 e 10), partos cesáreos, mães adolescentes (idade inferior a 20 anos), mães com escolaridade além do ensino médio e, de forma menos marcante, os partos prematuros (idade gestacional inferior a 37 semanas) (d'Orsi \& Carvalho, 1998).

A avaliação realizada dos dados brasileiros produzidos pelo SINASC, após seis anos de implantação no país, indicou que a cobertura se encontrava, em 1996, em torno de $85 \%$ para o país como um todo. As variáveis que apresentaram qualidade excelente/boa de preenchimento são sexo, peso ao nascer, duração da gestação, tipo de gravidez, tipo de parto e idade da mãe. A variável "Índice de Apgar", com elevado grau de não preenchimento, indica que, em muitas áreas, esta medida não é realizada (Mello-Jorge et al., 1996).

Estudo de validação do SINASC no Estado de São Paulo, em 1992, demonstrou, através da comparação das DNs com os prontuários hospitalares das mães, uma excelente cobertura do SINASC $(99,5 \%)$ e boa fidedignidade para quase todas as variáveis, exceto índice de Apgar e nível de instrução da mãe (Mello-Jorge et al., 1993). A informação sobre assistência pré-natal apresenta percentuais elevados de ausência de informação (28\%), inviabilizando a análise (Silva, 1996).

Ainda que hoje, no Município do Rio de Janeiro, quase $100 \%$ dos nascimentos sejam hospitalares, a discussão do acesso às maternidades é pertinente. O sistema de saúde no país, apesar de ressaltar a necessidade de integração entre os três períodos de assistência (pré-natal, parto e puerpério), nunca, efetivamente, viabilizou essa integração. Esse fato faz com que a assistência no período pré-natal seja feita sem nenhuma retaguarda hospitalar. Além disto, a impossibilidade de acesso às informações, de forma contínua e integral, em qualquer um destes períodos, fragiliza a assistência, expondo a puérpera e o recém-nato a riscos de vida (Tanaka, 1995).

Segundo dados da Secretaria Municipal de Saúde do Rio de Janeiro (1994), o município sofreu, nos últimos anos, uma redução de leitos obstétricos públicos, causada, principalmente, pela menor participação das instituições estaduais e federais no volume de internações obstétricas, em virtude da carência de recursos humanos, especialmente enfermeiras e auxiliares de enfermagem. Essa sobrecarga dos serviços públicos municipais levou ao aumento da utilização de serviços privados que têm convênio com o SUS, alguns de qualidade questionável. O pagamento do parto é feito por procedimento, sendo o valor atribuído pelo SUS muito inferior aos gastos necessários para uma assistência adequada, o que resulta em assistência desqualificada.

Outra questão a ser levantada é a desigualdade na oferta dos serviços de saúde no município, entre as Áreas de Planejamento (AP) que o compõe. O déficit de leitos públicos é maior nas regiões mais periféricas, não só em relação ao atendimento de gestantes saudáveis, mas também, e principalmente, para gestantes e recém-natos com patologias que exijam atendimentos mais complexos, apesar de já existir substancial incorporação de tecnologias sofisticadas e de alto custo à atenção pré-natal e perinatal. Como agravante, alguns municípios 
próximos não possuem rede de serviços de saúde adequada, em especial maternidades, sobrecarregando a rede do Município do Rio de Janeiro. Esses fatos, ainda que não tenham provocado alterações nas coberturas de partos hospitalares, originaram uma verdadeira peregrinação de gestantes e recém-natos em busca de assistência adequada (Gomes, 1995).

Vários fatores atuam como responsáveis pelo excesso de partos operatórios, podendo ser divididos em fatores relacionados à assistência médica e fatores sócio-culturais. O primeiro grupo passa por questões como uma formação fragilizada do médico para acompanhar com segurança a evolução de um parto normal e para realizar um parto espontâneo em gestantes que já sofreram uma cesárea, além da comodidade de o médico programar o seu horário de trabalho. Já o segundo grupo passa por questões ligadas à gestante, como o medo da dor do parto espontâneo e da deformação do corpo, assim como a opção da realização concomitante da laqueadura tubária.

O padrão sócio-econômico é um dos fatores que seleciona a demanda dos serviços de saúde e aponta para a dicotomia entre o setor público e o setor privado. No público, dificilmente se estabelece um vínculo entre a gestante e o sistema de saúde, levando a um desconhecimento da história da gestação e, em razão da rotina de carga de trabalho nessas instituições, a uma tendência de se intervir precocemente na evolução dos trabalhos de parto, a fim de que estes não se prolonguem para o plantão seguinte. Já no setor privado (sem convênio), ao contrário, a gestante é acompanhada no período pré-natal pelo médico que realizará o seu parto, tendo a oportunidade de estabelecer uma relação prévia de confiança. Entretanto, as altas taxas de cesáreas demonstram que isso não garante uma condução mais adequada para o parto.

\section{Material e métodos}

Os dados de nascimentos são provenientes do Sistema de Informações sobre Nascidos Vivos (SINASC), disponibilizados pela Coordenação de Programas de Epidemiologia/Sub-Gerência de Dados Vitais da Secretaria Municipal de Saúde (SMS/RJ), para o ano de 1995. O mapa digital de bairros e regiões administrativas foi obtido, agregando-se setores censitários digitalizados e disponibilizados pelo Departamento de Informações para a Saúde/Centro de Informação Científica e Tecnológica (CICT)/Fundação Oswaldo Cruz (FIOCRUZ). As variáveis referen- tes aos hospitais são provenientes da Pesquisa sobre Assistência Médico-Sanitária (AMS) / IBGE para o ano de 1992, disponibilizadas pelo Departamento de Informações para a Saúde/ CICT/FIOCRUZ.

Para a presente investigação, os seguintes indicadores foram selecionadas a partir do trabalho de d'Orsi \& Carvalho (1998): proporção de nascidos vivos com Apgar bom (Apgar no primeiro minuto entre 8 e 10); de nascidos vivos com Apgar grave (Apgar no primeiro minuto entre 0 e 3); de nascidos vivos com Apgar ignorado (sem preenchimento do campo Apgar no primeiro minuto); de nascidos vivos com baixo peso (inferior a $2.500 \mathrm{~g}$ ); de prematuros (idade gestacional inferior a 37 semanas); de partos cesáreos; de mães adolescentes (idade inferior a 20 anos); de mães com idade igual ou superior a 35 anos; de mães com escolaridade igual ou superior ao ensino médio; e de mães com escolaridade ignorada. A descrição do perfil das maternidades foi feita utilizando os recursos gráficos do pacote estatístico S-Plus.

$\mathrm{Na}$ análise do perfil de nascidos vivos e mães por unidade de saúde, do total de 134 estabelecimentos, optou-se por excluir 35 hospitais que registraram menos de 50 nascimentos no ano de 1995, por apresentarem flutuação aleatória dos indicadores, sendo, então, analisados 99 estabelecimentos. As 25 maternidades com mais de mil partos anuais foram analisadas mais detalhadamente.

Os hospitais foram classificados em análise de aglomerados multivariada pelo algoritmo $k$ means (Hartigan,1975), no pacote estatístico $S A S$. O peso das variáveis na construção dos grupos foi avaliado segundo o indicador R2/ (1-R2), onde $\mathrm{R}^{2}$ mede a variância entre os grupos e (1-R2) mede a variância intragrupos. A análise de aglomerados das unidades de saúde foi construída baseada em seis indicadores: proporção de mães adolescentes, proporção de mães com escolaridade igual ou superior ao ensino médio, proporção de prematuros, proporção de partos cesáreos, proporção de baixo peso e proporção de Apgar grave no primeiro minuto.

Para o mapeamento do fluxo de gestantes da residência para a maternidade foi utilizado o programa ARROWS do software MAPINFO. A unidade de análise foi a região administrativa, sendo excluídos os fluxos menores que cinqüenta nascimentos. A largura de cada flecha é proporcional ao fluxo de gestantes. Utilizou-se uma cruz como símbolo para a localização aproximada dos vinte cinco principais hospitais, coloridas conforme a classificação multivariada. 


\section{Resultados}

O Sistema de Informações sobre Nascidos Vivos (SINASC) registrou 107.157 nascimentos no ano de 1995, dos quais 98.409 ocorridos em estabelecimentos de saúde do Município do Rio de Janeiro. $\mathrm{O}$ índice de não-preenchimento das variáveis, em geral, foi baixo, variando de $0,3 \%$ para "tipo de parto" a 5,4\% para "instrução da mãe". As variáveis "número de filhos nascidos vivos", "número de filhos nascidos mortos" e "pré-natal” foram excluídas da análise pelo alto índice de não-resposta, impossibilitando o estudo da paridade, fato também verificado em outros trabalhos (d'Orsi \& Carvalho, 1998).

Na última coluna da Tabela 1, são apresentados os indicadores para os nascimentos ocorridos em estabelecimentos localizados no Município do Rio de Janeiro. Destaca-se o alto percentual de partos cesáreos $(46,0 \%)$ e de mães adolescentes (18,2\%). Quanto à escolaridade, $34,1 \%$ das mães tinham cursado o ensino médio ou mais. Essa variável apresenta percentual relativamente alto de "ignorado" (5,7\%). As três maiores maternidades realizaram, cada uma, acima de seis mil partos anuais.

Analisando os hospitais com mais de mil nascimentos, observou-se que os indicadores situam-se, freqüentemente, nos extremos máximo e mínimo (percentil 90 e 10 ou quartis). Nestes hospitais é grande o intervalo de distribuição de "partos cesáreos" (9,8\% a 84,5\%), "mães com escolaridade além do ensino médio" (5,2\% a 93,1\%), “Apgar bom” (0,3\% a 82,8\%) e "Apgar ignorado" (0,4\% a 84,4\%). Já "baixo peso" (4,6\% a $29,2 \%)$, "prematuros" $(0,2 \%$ a 29,9\%), "mães adolescentes" (1,8\% a 25,3\%), "mães com idade acima de 35 anos" (6,2\% a $17,4 \%)$, "Apgar grave" (0\% a 10\%) e "mães com escolaridade ignorada” $(0,1 \%$ a $41,1 \%)$ possuem amplitude menor.

Níveis elevados de não-preenchimento impedem uma análise adequada dos resultados. Por exemplo, na casa de saúde de maior volume de partos, em virtude de um altíssimo índice de Apgar ignorado (84,4\%), ocorre uma distorção nas proporções de Apgar bom (0,3\%) e de Apgar grave $(0,0 \%)$, e isso se torna mais evidente quando se compara com a maternidade em segundo lugar em volume de partos, que, com uma clientela de perfil semelhante, apresenta índices bem diferentes para essas proporções. Em relação à escolaridade, chama a atenção uma maternidade recentemente municipalizada com aproximadamente metade das DNs (41\%) emitidas com esse campo nãopreenchido.

Outro resultado inusitado é o fato de quatro hospitais apresentarem proporções de "prematuros" maior que "baixo peso", evidenciando um erro de preenchimento, na medida em que o evento baixo peso resulta tanto da prematuridade quanto do retardo de crescimento intra-uterino, portanto devendo ser sempre maior que a proporção de prematuros.

A Figura 1 apresenta os boxplots dos indicadores por hospitais relativos ao recém-nato e à mãe. Em relação às características do recém

\begin{tabular}{|c|c|c|c|c|}
\hline & \multicolumn{3}{|c|}{ Clustera } & \multirow[t]{2}{*}{ Total } \\
\hline & A & $\mathrm{B}$ & $\mathrm{R}^{2}\left(1-\mathrm{R}^{2}\right)$ & \\
\hline Estabelecimentos & 69 & 30 & - & \\
\hline Número de nascidos vivos & 30.143 & 67.740 & - & $98.409 b$ \\
\hline \multicolumn{5}{|l|}{ Proporções } \\
\hline Cesárea & $81,5 \%$ & $32,2 \%$ & 3,43 & $46,0 \%$ \\
\hline Baixo peso & $6,6 \%$ & $12,6 \%$ & 0,41 & $10,7 \%$ \\
\hline Prematuros & $4,7 \%$ & $9,6 \%$ & 0,21 & $7,6 \%$ \\
\hline Mãe adolescente & $8,2 \%$ & $20,8 \%$ & 1,83 & $18,2 \%$ \\
\hline Apgar grave & $1,4 \%$ & $3,0 \%$ & 0,26 & $2,5 \%$ \\
\hline Escolaridade > ensino médio & $62,7 \%$ & $17,6 \%$ & 1,79 & $34,1 \%$ \\
\hline
\end{tabular}

a Excluídos os estabelecimentos com menos de cinqüenta nascimentos anuais.

b Todos os nascimentos ocorridos no Município do Rio de Janeiro. 
nascido, as proporções de baixo peso, prematuros, Apgar grave e Apgar ignorado apresentam distribuição concentrada, com mediana inferior a 0,10 e um grande número de valores marginais no extremo superior. Em relação às características da mãe e ao tipo de parto, as proporções de mães com idade igual ou superior a 35 anos e escolaridade ignorada apresentam um padrão de distribuição semelhante ao das características do recém nascido. Em oposição, as proporções de partos cesáreos e de mães com escolaridade igual ou superior ao ensino médio apresentam grande amplitude de variação. A mediana para a proporção de partos cesáreos foi extremamente alta, um pouco inferior a 0,80 , e para a proporção de mães com escolaridade igual ou superior ao ensino médio foi um pouco acima de 0,50. Já a proporção de mães adolescentes apresenta uma amplitude menor, com a mediana em torno de 0,10 . Esses três últimos indicadores são, portanto, mais úteis para discriminar diferenças entre unidades de saúde.

Na Tabela 1 estão apresentados os principais resultados da análise de cluster. O grupo A representa a maioria das instituições (69), porém não a maioria dos recém-nascidos (30.143), ou seja, a grande parte de suas unidades de saúde são de menor porte. Já o grupo B representa a minoria das instituições (30), porém a maioria dos recém-nascidos (67.740), aglutinando as grandes maternidades. Em relação aos indicadores selecionados, os mais importantes na diferenciação dos grupos foram as proporções de partos cesáreos, mães adolescentes e mães com escolaridade igual ou superior ao ensino médio.

No grupo A, é elevada a proporção de partos operatórios $(0,81)$, sendo a clientela constituída por mulheres com nível de escolaridade igual ou superior ensino médio $(0,63)$ e com baixa proporção de mães adolescentes $(0,08)$. Em relação aos recém-nascidos, verifica-se baixa proporção de baixo peso $(0,07)$, prematuridade $(0,05)$ e Apgar grave $(0,01)$. Esses resultados, quando comparados ao número excessivo de partos cesáreos, sugerem não haver relação com sofrimento fetal.

Já o grupo B tem como características o predomínio do parto espontâneo, ainda que, baseado em padrão de outros países, a freqüência de parto operatório ainda seja elevada $(0,32)$. Além disso, há um percentual elevado de mães adolescentes $(0,20)$ e baixo nível de escolaridade $(0,18)$. Quanto aos recém-nascidos, nota-se um percentual relativamente elevado de baixo peso ao nascer $(0,13)$, prematuros $(0,10)$ e Apgar grave $(0,03)$.
Figura 1

Distribuição de indicadores por estabelecimento de assistência ao parto relativos às condições da gestante e do recém-nato. Município do Rio de Janeiro, 1995.
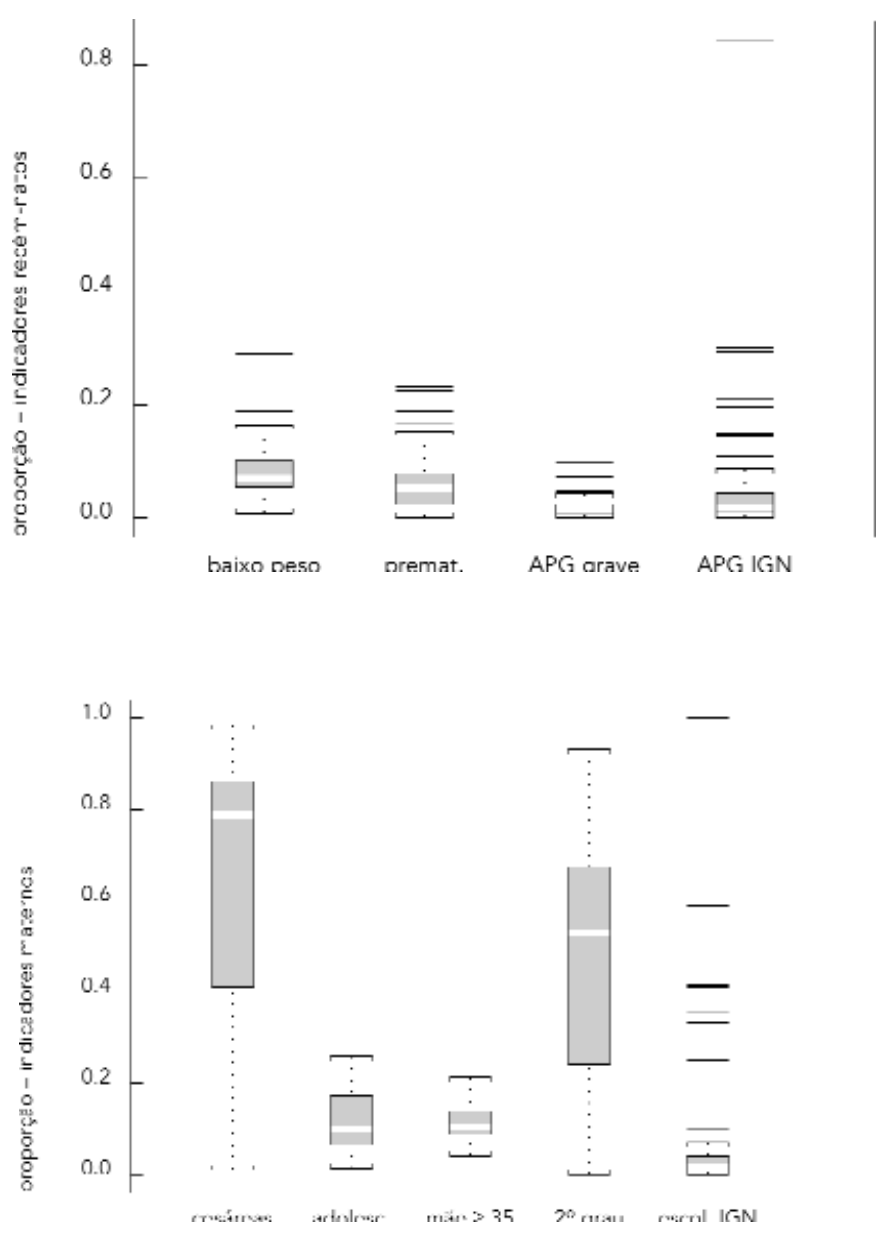

A Figura 2 apresenta os boxplots de alguns indicadores aqui analisados, separados pelos grupos originados da análise de cluster. A mediana do total de nascidos vivos para o grupo A foi muito inferior a quinhentos nascimentos (maternidades de pequeno porte); já para o grupo B, foi superior a 1.500 (maternidades de grande porte). As medianas para os indicadores relacionados à mãe e ao tipo de parto foram bem distintas entre os grupos de maternidades. Para a proporção de partos cesáreos, o grupo A apresentou uma mediana considerada muito elevada, superior a 0,80 , com valores marginais no extremo inferior da distribuição (todos acima de 0,40 ), e o grupo B, proporção inferior a 0,30. A proporção de mães com es- 
Figura 2

Distribuição de indicadores por estabelecimento de assistência ao parto, segundo classificação multivariada.

Município do Rio de Janeiro, 1995.
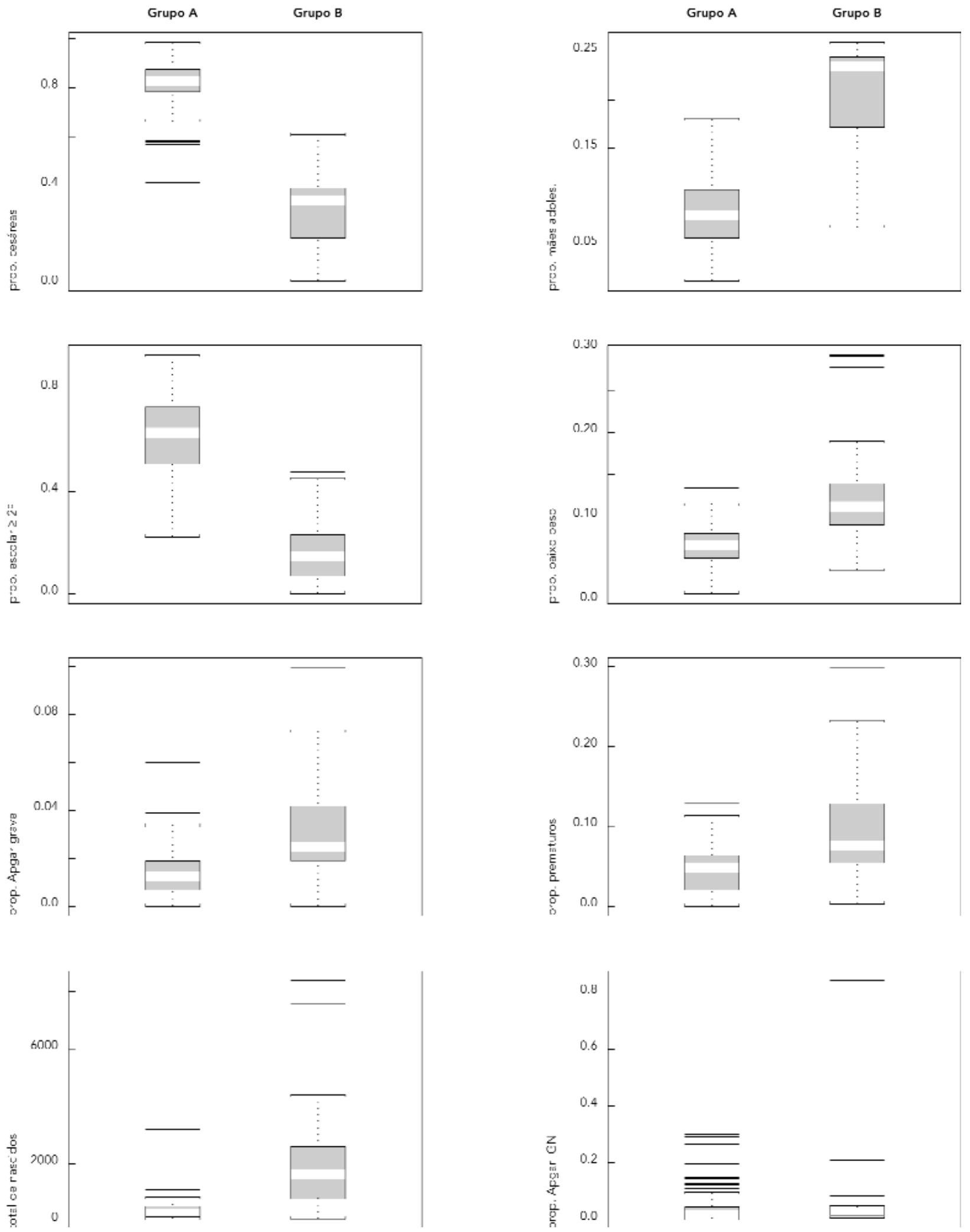
colaridade igual ou superior ao ensino médio no grupo A foi superior a 0,60 e, no grupo B, muito baixa, inferior a 0,20 . A proporção de mães adolescentes foi no grupo A em torno de 0,08 e no grupo B, um pouco inferior a 0,25. Em contrapartida, para os indicadores das condições do recém-nascido, as diferenças nos grupos foram menos marcante. A proporção de baixo peso apresenta a mediana um pouco acima de 0,05 no grupo A e em torno de 0,10 no grupo B. A distribuição é mais concentrada no grupo A para a proporção de prematuros e para a proporção de Apgar grave, ficando a mediana em valores próximos. A proporção de Apgar ignorado apresenta distribuição semelhante nos dois grupos, com um grande outlier no grupo $B$ : a casa de saúde de maior volume de partos, onde $84 \%$ das decla- rações de nascidos vivos não tiveram esta variável preenchida.

Na Tabela 2 foi feita a análise dos estabelecimentos, comparando-se os grupos gerados segundo variáveis selecionadas da AMS. Podese observar que não há distinção entre os grupos, ou seja, em ambos a maior parte $(70 \%)$ dos estabelecimentos é privada, do tipo sociedade com fins lucrativos. Utilizando o mesmo critério de outros trabalhos, os estabelecimentos foram classificados segundo dois segmentos denominados SUS e Não-SUS. O primeiro grupo é composto por todos os estabelecimentos públicos e os privados que têm convênio com o SUS, e os restantes estão no segundo grupo. Seguindo essa classificação, nota-se que a maior parte $(60 \%)$ das maternidades situa-se fora da gestão do SUS (não significando a maior parte

Tabela 2

Indicadores selecionados da AMS segundo Classificação Multivariada dos Estabelecimentosa.

Município do Rio de Janeiro, 1995.

\begin{tabular}{|c|c|c|c|c|c|c|}
\hline \multirow[t]{2}{*}{ Indicador } & \multicolumn{2}{|c|}{ A } & \multicolumn{2}{|c|}{ B } & \multicolumn{2}{|c|}{ Total } \\
\hline & $\mathrm{n}$ & $\%$ & $\mathrm{n}$ & $\%$ & $\mathrm{n}$ & $\%$ \\
\hline \multicolumn{7}{|l|}{ Constituição jurídica } \\
\hline Federal & 7 & 10,6 & 3 & 10,0 & 10 & 10,4 \\
\hline Estadual & 9 & 13,7 & 0 & 0,0 & 9 & 9,4 \\
\hline Municipal & 2 & 3,0 & 5 & 16,7 & 7 & 7,3 \\
\hline Privada & 48 & 72,7 & 22 & 73,3 & 70 & 72,9 \\
\hline Total & 66 & 100,0 & 30 & 100,0 & 96 & 100,0 \\
\hline \multicolumn{7}{|l|}{ Natureza jurídica } \\
\hline Órgão público & 14 & 21,2 & 7 & 23,2 & 21 & 21,9 \\
\hline Autarquia & 2 & 3,0 & 0 & 0,0 & 2 & 2,1 \\
\hline Fundação & 1 & 1,5 & 1 & 3,4 & 2 & 2,1 \\
\hline Empresa pública & 1 & 1,5 & 0 & 0,0 & 1 & 1,0 \\
\hline Sociedade s/ lucro & 6 & 9,2 & 1 & 3,4 & 7 & 7,3 \\
\hline Sociedade c/ lucro & 42 & 63,6 & 21 & 70,0 & 63 & 65,6 \\
\hline Total & 66 & 100,0 & 30 & 100,0 & 96 & 100,0 \\
\hline \multicolumn{7}{|l|}{ Cadastro INSS } \\
\hline SUS & 29 & 56,0 & 10 & 66,7 & 39 & 60,0 \\
\hline Não-SUS & 37 & 44,0 & 20 & 33,3 & 57 & 40,0 \\
\hline Total & 66 & 100,0 & 30 & 100,0 & 96 & 100,0 \\
\hline \multicolumn{7}{|l|}{ Localização (AP) } \\
\hline 1.0 & 15 & 23,4 & 6 & 20,0 & 21 & 22,3 \\
\hline $2.1+2.2$ & 10 & 15,6 & 5 & 16,7 & 15 & 16,0 \\
\hline $3.1+3.2+3.3$ & 31 & 48,4 & 14 & 46,6 & 45 & 47,9 \\
\hline 4.0 & 4 & 6,3 & 2 & 6,7 & 6 & 6,4 \\
\hline $5.1+5.2+5.3$ & 4 & 6,3 & 3 & 10,0 & 7 & 7,4 \\
\hline MRJ & 64 & 100,0 & 30 & 100,0 & 94 & 100,0 \\
\hline
\end{tabular}

a Excluídos os estabelecimentos com menos de cinqüenta nascimentos anuais e os ignorados para cada categoria. 
dos nascimentos), diferentemente da Região Sudeste, onde esse percentual é somente $30 \%$ (Viacava \& Bahia, 1996). Este resultado não varia quando se analisa segundo os grupos A e B. Não foi possível estimar o número de leitos obstétricos dentro dos estabelecimentos de especialidades mistas, nem tampouco estabelecer os pertencentes ao segmento SUS e NãoSUS, em função da ausência desse nível de detalhamento nos dados da AMS. A localização dos hospitais nas APs do município não aponta diferença entre os dois grupos A e B. Ou seja, nesse nível de análise, a caracterização das maternidades a partir da proporção de partos cesáreos e de características das gestantes, variáveis que tiveram maior peso na construção dos grupos, não apresenta relação com as variáveis que caracterizaram os estabelecimentos na AMS.

A Figura 3 permite identificar os fluxos entre a região administrativa (RA) de residência da mãe e a RA da maternidade onde ocorreu o nascimento, além de localizar e caracterizar os principais estabelecimentos, segundo sua classe. É possível observar maior concentração de serviços nos bairros do Centro e Zona Sul. A maior freqüência do grupo B entre as vinte e cinco maiores maternidades reflete a composição deste grupo, que concentra os grandes serviços. O sentido do fluxo de nascimentos no município é da Zona Oeste para a Zona Central, confirmando claramente que as APs onde há disponibilidade de serviços de saúde possuem um poder de atração em relação aos nascimentos.

\section{Discussão e conclusões}

As variáveis que melhor distinguiram as principais maternidades no Município do Rio de Janeiro foram as proporções de mães com escolaridade igual ou superior ao ensino médio, mães adolescentes e partos cesáreos, ou seja, os indicadores relacionados às características da mãe e ao tipo de parto foram os mais eficazes em discriminar as unidades de saúde. $\mathrm{O}$ programa de classificação multivariada foi rodado diversas vezes, alterando variáveis e parâmetros de convergência num processo interativo. Nenhuma das demais configurações testadas permitiu classificar de forma consistente as maternidades.

Dois tipos de maternidades foram identificados através destes indicadores: um com grande número de partos cesáreos e boas condições da parturiente e recém-nato, o que é aparentemente contraditório, sendo a escolari- dade da mãe mais alta e os hospitais menores. O outro, com maior proporção de partos normais e indicadores que apontam riscos do recém-nascido, é constituído por grandes fábricas de partos, onde as gestantes mais jovens e de menor escolaridade são atendidas.

Diversos estudos revelam que o Brasil apresenta uma elevada taxa de partos por cesariana (42\%). Estudo realizado em São Paulo mostrou que, do total de partos realizados, $46,2 \%$ eram cesáreas (Tanaka, 1989), freqüência considerada elevada segundo critérios da Organização Mundial da Saúde (OMS). Na Inglaterra, onde a taxa de cesárea é de $13 \%$ a $15 \%$, tem-se observado um aumento do número de partos cesáreos, que em algumas regiões chega a 25\% (Umberlino, 1996). Estudo realizado em Pelotas (RS), em 1989, demonstrou uma freqüência maior de partos cesáreos (47\%) em famílias de maior renda que nas de baixa renda, e, no Rio de Janeiro, evidenciou-se maior freqüência de cesáreas em gestantes residentes nos bairros mais ricos da cidade (d'Orsi \& Carvalho, 1998). Além disso, os partos cesáreos foram mais freqüentes nas gestações de baixo risco (33\%) do que nas de alto risco (26\%) (Victora et al., 1989).

Sabe-se, também, da indicação do parto cesáreo para a realização concomitante da laqueadura tubária. Isso nos leva a apontar para a dicotomia entre o setor público e o setor privado, em que, no público, o atendimento se dá em maternidades de esfera municipal, quando dificilmente se estabelece um vínculo entre a gestante que busca atendimento e o sistema de saúde. Já no setor privado, ao contrário, a gestante é acompanhada no período pré-natal pelo médico que realizará o seu parto, tendo a oportunidade de estabelecer uma relação prévia de confiança, o que, entretanto, não garante uma condução mais adequada do parto. Segundo a OMS, o percentual de partos operatórios não deve ultrapassar $20 \%$ do total de partos, e sua realização deve obedecer a critérios clínicos da gestante e do feto que contra-indiquem o parto espontâneo. O parto operatório apresenta riscos inerentes ao ato cirúrgico, além de implicar um maior tempo de recuperação para a puérpera. Este estudo confirmou, portanto, o abuso por parte dos profissionais médicos na realização do parto operatório, muito mais freqüente do que o recomendado pela OMS, principalmente nas unidades privadas, reforçando a necessidade de avaliação dessas intervenções.

A AMS acrescentou poucas informações relevantes para a análise das maternidades. As categorias "privado" e "com fins lucrativos" reúnem, na verdade, estabelecimentos bem dife- 
Mapa de fluxo de gestantes entre RA de residência e RA do parto e localização dos 25 estabelecimentos com maior número de partos anuais. Município do Rio de Janeiro, 1995.
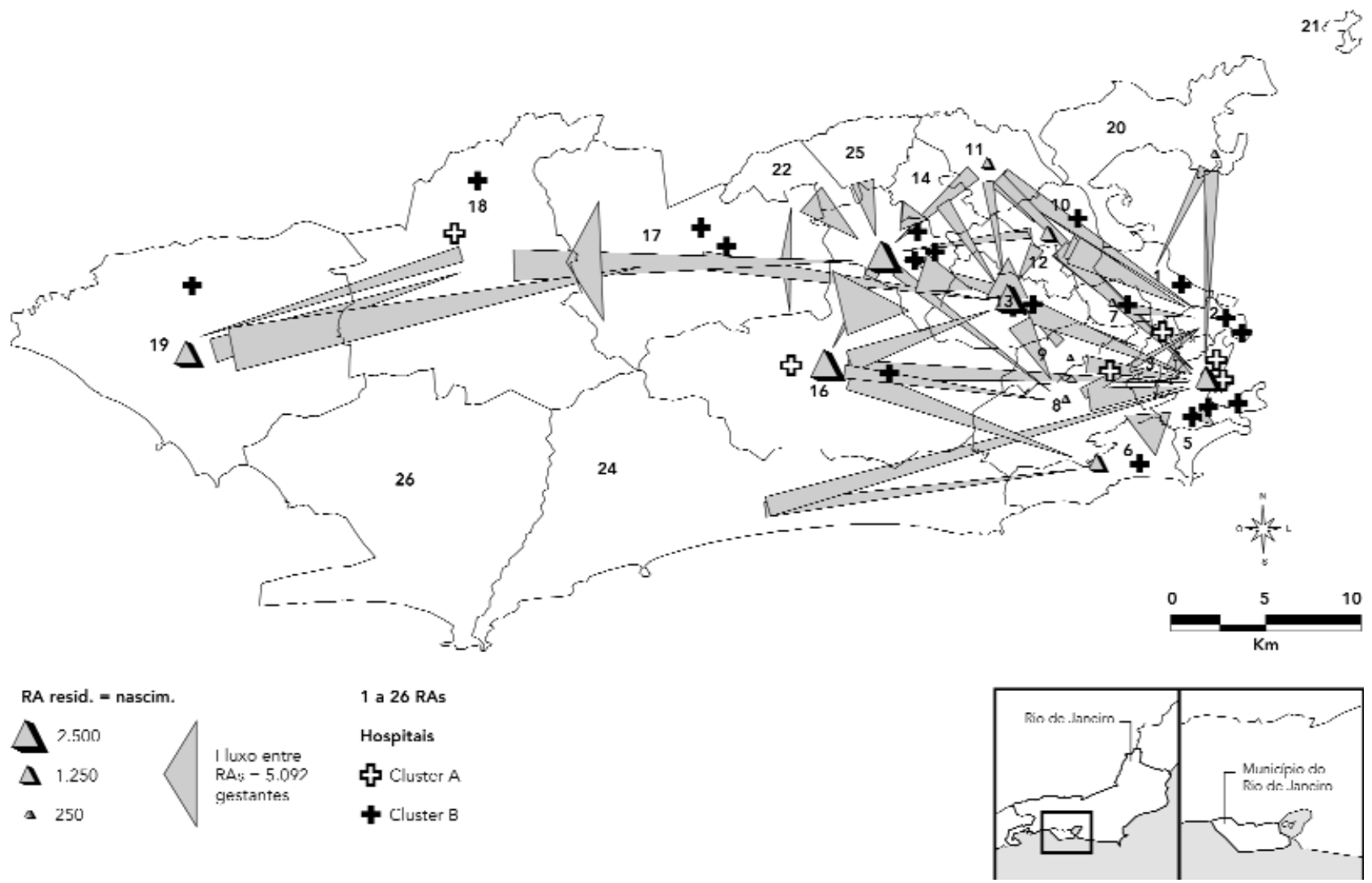

rentes: aqueles conveniados com o SUS, voltados para o atendimento à população de baixa renda, e outros que atendem convênios privados e pacientes particulares. A informação do número de leitos obstétricos dentro de cada estabelecimento, pertencentes ou não ao segmento SUS, permitiria refinar esta análise.

Em geral, os fluxos das gestantes apontam no sentido das regiões mais ricas da cidade, ligando as parturientes que residem em áreas de baixa renda (Zona Oeste) a áreas onde se encontram melhores e maiores ofertas de serviços de saúde. Observa-se que são grandes as distâncias percorridas. Uma boa distribuição geográfica apresentaria um padrão de setas curtas entre regiões administrativas vizinhas.

O mapeamento do fluxo permitiu traçar o perfil de migração das gestantes no momento do parto e o perfil de oferta de serviços de saú- de distribuídos pelas regiões administrativas do município. É importante citar que, na verdade, o fluxo da maioria das gestantes atendidas em grande parte das maternidades do grupo B não se deu de forma tão linear como nas setas que se vê no mapa. Infelizmente, não há software que consiga dar conta do mapa da peregrinação das gestantes em busca de um local para dar à luz. 


\section{Referências}

CAPER, P., 1987. The epidemiologic surveillance of medical care. American Journal of Public Health, 77:669-670.

D'ORSI, E. \& CARVALHO, M. S., 1998. Perfil de nascimentos no Município do Rio de Janeiro. Cadernos de Saúde Pública, 14:367-379.

GOMES, M. A. S. M., 1995. Aspectos da Qualidade do Atendimento à Gestação e ao Parto através da Percepção das Usuárias. Dissertação de Mestrado, Rio de Janeiro: Instituto Fernandes Figueira, Fundação Oswaldo Cruz.

HARTIGAN, J. A., 1975. Clustering Algorithms. New York: John Wiley \& Sons Inc.

MÉDICI, A. C.; MACHADO, M. H.; NOGUEIRA, R. P. \& SÁBADO, N. G., 1992. Mercado de Trabalho em Saúde no Brasil: Estrutura e Conjuntura. Rio de Janeiro: Secretaria de Desenvolvimento Educacional, Escola Nacional de Saúde Pública.

MELLO-JORGE, M. H. P.; GOTLIEB, S. L. D.; SOBOLL, M. L. M. S.; BALDIJÃO, M. F. A. \& LATORRE, M. R. D. O., 1993. Avaliação do sistema de informação sobre nascidos vivos e o uso de seus dados em epidemiologia e estatísticas de saúde. Revista de Saúde Pública, 27:2-46.
MELlO-JORGE, M. H. P.; GOTLIEB, S. L. D. \& OLIVEIRA, H., 1996. O sistema de informação sobre nascidos vivos: Primeira avaliação dos dados brasileiros. Informe Epidemiológico do SUS, 5:1548.

SMS/RJ (Secretaria Municipal de Saúde do Rio de Janeiro), 1994. Assistência Obstétrica e Neonatal no Município do Rio de Janeiro. Rio de Janeiro: SMS/RJ.

SILVA, R. I., 1996. Perfil de nascimentos no Município do Rio de Janeiro - 1995. Saúde em Foco, 5:28-31.

TANAKA, A. C. A., 1995. Maternidade: Dilema entre Nascimento e Morte. São Paulo: Editora Hucitec/ ABRASCO.

TANAKA, A. C. A.; SIQUEIRA, A. A. F. \& BAFILE, P. N., 1989. Situação de saúde materna e perinatal no Estado de São Paulo, Brasil. Revista Saúde Pública, 23:67-75.

UMBERLINO, M., 1996. O modelo de assistência a gestação, parto e nascimento na Inglaterra. Saúde em Foco, 5:15-17.

VIACAVA, F. \& BAHIA, L., 1996. Os serviços de saúde segundo o IBGE. Dados/FIOCRUZ, 20:3-30.

VICTORA, C. G.; BARROS, F. C. \& VAUGHAN, J. P., 1989. Epidemiologia da Desigualdade. São Paulo: Editora Hucitec. 